山्山FANÇAISE

$>\mathrm{DE}$

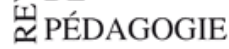

\section{Revue française de pédagogie}

Recherches en éducation

$196 \mid 2016$

Apprendre à lire et à écrire au cours préparatoire : enseignements d'une recherche collective

\title{
L'alternance mise en œuvre par les enseignants universitaires : le paradoxe de la coupure et du lien dans les licences professionnelles
}

Work-linked training implemented by university teachers: the paradox of

linkage and separation in vocational degrees

Maryse Bournel-Bosson et Muriel Henry

\section{(2) OpenEdition}

Journals

Édition électronique

URL : https://journals.openedition.org/rfp/5094

DOI : 10.4000/rfp.5094

ISSN : 2105-2913

Éditeur

ENS Éditions

Édition imprimée

Date de publication : 30 septembre 2016

Pagination : 117-128

ISSN : 0556-7807

Référence électronique

Maryse Bournel-Bosson et Muriel Henry, «L'alternance mise en œuvre par les enseignants

universitaires : le paradoxe de la coupure et du lien dans les licences professionnelles », Revue

française de pédagogie [En ligne], 196 | 2016, mis en ligne le 30 septembre 2019, consulté le 26 janvier

2022. URL : http://journals.openedition.org/rfp/5094 ; DOI : https://doi.org/10.4000/rfp.5094 


\title{
L'alternance mise en œuvre par les enseignants universitaires : le paradoxe de la coupure et du lien dans les licences professionnelles
}

\author{
Maryse Bournel-Bosson \\ Muriel Henry
}

\begin{abstract}
Dans le cas particulier des licences professionnelles, cet article investigue les effets de la mise en œuvre de l'alternance sur les processus pédagogiques universitaires. Les entretiens réalisés avec les enseignants, qui constituent la source des données, mettent en exergue les rapports entre université et milieu professionnel. L'analyse fine des discours montre la dimension paradoxale de ces rapports, faite de "coupure et de lien », et ses réalisations diverses : si la visée d'insertion des étudiants constitue un lien entre les enseignants universitaires et les milieux professionnels, la régulation des rythmes entre les deux espaces en revanche fait tension. L'évaluation des acquis des étudiants s'inscrit pour sa part nettement dans ce qui fait coupure.

Mots-clés (TESE) : université, licence, formation en alternance, formation et enseignement professionnels
\end{abstract}

En 2007, I'université s'est vu confier en France une mission explicite d'insertion professionnelle (loi dite LRU'). Cette prescription a réactivé le mouvement de la professionnalisation des études supérieures engagé depuis plusieurs décennies. La finalité est d'accroître l'efficacité du système universitaire en le rapprochant du «système productif » (Maillard, Veneau \& Grandgérard, 2004, p. 7) ou, dit autrement, d'identifier en quoi

1 Loi relative aux libertés et responsabilités des universités. les savoirs académiques peuvent prendre sens au regard de problématiques dites de terrain. Ce mouvement implique des changements importants dans le quotidien de l'activité des acteurs de l'université, notamment dans les dimensions de conception et de mise en œuvre des dispositifs de formation. Si l'université n'est donc plus (ou pas) seulement un lieu de transmission des savoirs académiques ou théoriques, une redéfinition des rapports entre les lieux professionnels et les lieux de formation est à opérer, liée à une attention spécifique à l'ingénierie de formation et à la 
pédagogie. L'enjeu social est majeur et il suppose une mobilisation de tous les acteurs de la communauté universitaire qui dès lors s'engagent dans un développement de leurs activités. Dans ce vaste chantier, cet article vise à investiguer la manière dont les responsables pédagogiques, enseignants-chercheurs, inventent cette professionnalisation qui ne peut se résumer à un rapport mécaniste entre formation et emploi. Des travaux de recherche existent à ce sujet notamment au niveau de la mise en œuvre d'une approche par les compétences (Chauvigné \& Coulet, 2010; Lemistre, 2015), mais c'est sous I'angle de l'alternance ${ }^{2}$ que nous souhaiterions apporter un éclairage. Celle-ci constitue en effet une forme identifiée socialement de transition professionnelle qui s'est propagée en direction d'une population étendue et diversifiée en âge et en niveau de qualification. Désormais, comme le souligne Mayen, «quand on veut résoudre un problème de professionnalisation, on convoque I'alternance et l'expérience » (2007, p. 85). L'efficacité du dispositif est posée comme allant de soi. On peut cependant raisonnablement penser que la réalité est autrement plus complexe, d'autant qu'à l'université, l'expérience de la mise en œuvre de l'alternance est relativement récente.

Nous nous proposons d'en étudier les contours dans le cadre d'un dispositif de formation ciblé, à savoir la licence professionnelle (LP). En effet, dans le système français, elle constitue le dernier diplôme national en date créé dans le supérieur (1999) - le master professionnel apparu ensuite relevant quant à lui d'une transformation des DESS au moment du passage au LMD (Licence-master-doctorat; de 2003 à 2006) - et elle condense les attendus en matière de professionnalisation. Maillard et Veneau (2006) écrivent, en référence à l'arrêté relatif à sa création, que celle-ci «marque son originalité en radicalisant l'exigence des relations entre I'Université et l'entreprise » et en la cristallisant sous le terme unique de "partenariat avec les professionnels » qui doit s'appliquer «à l'intégralité du processus de création et de mise en œuvre des formations». Comment se réalise cette injonction à la professionnalisation du point de vue de l'activité des responsables de LP à l'université? Et plus spécifiquement, qu'en est-il de sa réalisation si l'on se place sur le plan des conditions de mise en œuvre de l'alternance? Les pratiques concrètes liées à celle-ci sont-elles à la hauteur des

2 Le mot est à entendre comme « une formation professionnelle en alternance». finalités attribuées aux LP en matière d'ouverture large au monde professionnel? Telles sont les questions qui structurent notre activité de recherche dans le cadre de ce texte.

Après avoir rappelé les attendus de l'alternance dite intégrative fondée autour du paradoxe de la «coupure et du lien » (Lerbet-Seréni, 1997), paradoxe qui nécessite une attention soutenue à l'activité d'ingénierie du dispositif de formation, nous en étudions la spécificité au niveau de la licence professionnelle. Une analyse thématique et lexicale du discours de 22 responsables de LP interviewés dans le cadre d'une recherche ${ }^{3}$ est proposée : elle permet d'éclairer la problématique de la coupure et du lien en rendant compte de l'activité des responsables de formation dans ses dimensions organisationnelles, relationnelles mais aussi évaluatives.

\section{L'alternance : " une conjugaison d'espaces séparés »}

Dans l'enseignement supérieur français, plusieurs rapports, lois, accords interprofessionnels, ont contribué, au cours du xxe siècle, à institutionnaliser l'alternance comme un mode majeur de professionnalisation. Lichtenberger (1995) propose de penser que la période qui s'étend des années 1960 aux années 1990 a vu émerger " un modèle français de l'alternance», modèle auquel on va demander de relever de grands défis : lutter contre l'échec scolaire, tenter de diminuer le chômage et élever le niveau de qualification notamment pour les jeunes, renouveler l'adéquation formation/ emploi et les modalités d'insertion des jeunes diplômés (Lemistre, 2015), battre en brèche la suprématie des savoirs académiques et développer ce qui relève de «apprendre autrement». D'abord voie réservée aux élèves peu friands du système scolaire général, l'alternance est devenue un outil majeur de la politique d'insertion des jeunes, avec une extension à tous les niveaux de formation ${ }^{4}$ : depuis les formations pour le

3 Cette recherche s'est déroulée dans le cadre d'un Bonus Qualité Recherche $(B Q R)$ validé par l'université de Franche-Comté et réalisé en partenariat avec l'université de Bourgogne. Le rapport dans son intégralité est consultable grâce au lien suivant: $<$ http://psychologie.univ-fcomte.fr/download/section-psychologie/document/ articles-rapports/texterapportprofessionnalisationversionfinale. pdf> (consulté le 18 janvier 2018).

4 Dans le supérieur, la principale loi est la loi dite Séguin du 23 juillet 1987 qui ouvre l'apprentissage à des niveaux de qualification supérieurs au CAP. 
public dit de bas niveau de qualification jusqu'aux formations de niveau l $(\mathrm{bac}+5)$, y compris dans le domaine des lettres ou des sciences humaines. Les formes prises par l'alternance dans l'enseignement supérieur sont très diverses, l'étudiant pouvant être lié à l'entreprise par une convention de stage mais aussi par un contrat de travail tel que le contrat d'apprentissage ou le contrat de professionnalisation ${ }^{5}$.

Le dénominateur commun de l'alternance est représenté par la mise en situation professionnelle de l'étudiant dans un milieu de travail avec un degré d'autonomie croissant, couplée à une formation dite en centre. Stricto sensu, l'alternance ne désigne rien d'autre qu'un va-et-vient entre des lieux, va-et-vient inscrit dans des temporalités aux configurations multiples (jours/semaines/mois). À un premier niveau, on se situe dans une forme de naturalité apparente, l'efficacité pédagogique du dispositif semblant "être garantie par la stricte dualité des places, des rôles» (Patroucheva \& Triby, 2010, p. 118). Plusieurs interrogations apparaissent toutefois lorsque l'on avance dans l'analyse : tout passage en entreprise ne serait pas forcément professionnalisant, tout comme d'ailleurs «l'acquisition de compétences opérationnelles peut aussi se faire en situation purement scolaire» (Rose, 2008, p. 54). Ulmann va elle aussi dans ce sens en attirant l'attention sur la nécessité de prendre en considération, avec le plus grand soin, "les contextes et les situations de travail proposés aux étudiants» (2012, p. 163), au-delà de la mise en place d'un cadre contractuel bien installé (désignation d'un tuteur professionnel, explicitation des missions confiées aux étudiants avec une progression pédagogique, engagement mutuel de l'entreprise et de l'université pour encadrer I'apprenant).

Sous le vocable alternance, on observe dans la littérature abondante et historiquement située ${ }^{6}$ des évolutions dans la dénomination sans que l'on sache toujours très bien si elles rendent compte de pratiques effectives ou si elles visent à les développer. Les recherches de définition ainsi que les différentes

5 Plusieurs distinctions existent entre l'alternance version stage et l'alternance version contrat de travail : la durée plus longue du temps sur le terrain dans le second cas que dans le premier, la nature des liens entre l'université et le terrain qui sont généralement moins structurés dans la version stage.

6 Voir à ce sujet, par exemple, l'ouvrage collectif de 1992 publié à la documentation française (AFPA, CEE, CNAM et INRP) et cité en bibliographie qui établissait une revue de littérature sur l'alternance à partir des publications des 20 années écoulées. modalités de mise en œuvre sont généralement formulées sur un mode d'opposition ou plus finement dans une logique de hiérarchisation. On identifie ainsi la classification suivante déjà décrite en 1979 par Bourgeon et reprise en 1999 par Geay et alii :

- une alternance présentée comme étant "fausse» ou «juxtapositive» dans laquelle les deux entités que sont l'enseignement et le stage cohabitent sans liens formalisés (Malglaive, 1994, p. 28) mise à part la présence d'une autorité tutélaire qui en garantit les conditions économiques de réalisation. Faire se succéder mécaniquement les situations est reconnu comme étant peu profitable du point de vue des apprentissages et générant une perte de sens pour les formés. Ici, comme le mentionnent Geay et alii, (1999, p. 113), c'est le poids de l'institué, très fort, qui s'impose aux acteurs organisant le dispositif uniquement de manière bureaucratique;

- une alternance "associative», dénommée également «approchée» ou encore "empirique», basée sur l'enchaînement des deux entités que sont le lieu de formation et l'entreprise, la seconde étant envisagée comme une mise en application des enseignements dispensés dans la première. C'est un modèle de type «applicationniste » pour faire le parallèle avec la classification de Vanhulle, Mottier-Lopez et Deum (2007, p. 11) dans lequel «la logique de l'offre de formation formelle prédomine» (Wittorski, 2012, p.6). Les liens plus interpersonnels entre les acteurs permettent de dépasser la lourdeur administrative mais ils restent précaires;

- une alternance «intégrative» ou "réelle» ou encore «interactive» et même "copulative» (Geay et al., p. 114, en référence à Bourgeon) qui voit se réunir dans un système d'intérêts convergents et complémentaires les acteurs de l'entreprise, ceux du centre de formation et le jeune lui-même. Des allers et retours réguliers entre les différents lieux permettent une mise en perspective des apprentissages, l'apprenant étant situé intentionnellement au centre du dispositif (Chaix, 1993). Dans ce modèle, la scission théorie-pratique se trouve largement dépassée pour décrire les modalités variées d'appropriation et d'investissement des savoirs eux-mêmes divers (savoirs incorporés, expérientiels, de références, d'experts, homologués, savants, disciplinaires et interdisciplinaires...). Plus exactement, ce sont les capacités des sujets «à traverser et dépasser les tensions générées par la multi-référentialité des savoirs offerts par l'alternance» (Vanhulle, MottierLopez \& Deum, 2007, p. 37) qui deviennent centrales. 
Les enjeux se situent dans cette forme d'alternance au niveau des capacités de l'équipe pédagogique à favoriser ce processus d'interpénétration entre les différents univers dans lesquels le jeune évolue tout en ne prétendant pas à une forme d'achèvement.

Si l'alternance est «structurellement une conjugaison d'espaces séparés» (Roucoules, 2004, p. 138) qui pourtant doivent être en interaction, comment ce paradoxe de la «coupure et du lien » (Lerbet-Seréni, 1997) trouve-t-il des formes de réalisation? Cette question a été travaillée jusqu'à ce jour sous l'angle des apprenants mais encore peu du point de vue des concepteurs de la formation. Quelle activité déploientils pour parvenir à développer, dans le cadre de l'alternance, cette interaction des espaces de formation? Quelles modalités de partenariat sont mises en œuvre avec les entreprises et en quoi les obligent-elles à quitter une zone de confort et à partager leur plein pouvoir sur l'espace éducatif?

C'est sur ces questions que nous nous focalisons en situant le propos dans un contexte précis, celui du dispositif des licences professionnelles.

\section{L'alternance vue à travers le prisme des licences professionnelles}

\section{Le contexte de mise en œuvre du diplôme}

Fléchées explicitement vers l'insertion professionnelle des jeunes sortants avec un niveau bac +3 de l'université, les LP ont rencontré dès leur création un vif succès et elles n'ont pas cessé de voir augmenter leur effectif?. Aujourd'hui, le diplôme comporte 173 mentions différentes. Les demandes d'ouverture ont été fortement soutenues par les présidences d'universités du fait notamment qu'elles correspondaient aux objectifs de professionnalisation inscrits dans les projets d'établissement. Elles ont trouvé aisément leur place dans le schéma européen des diplômes (LMD) en offrant une voie d'accès au niveau licence pour les étudiants sortant principalement de DUT et secondairement de BTS ${ }^{8}$. Par contre, les étudiants issus d'une deuxième

7 Les diplômés de licence professionnelle représentent en moyenne près de $29 \%$ des 161000 diplômés de licence.

8 DUT (Diplôme universitaire de technologie) et BTS (Brevet de technicien supérieur) sont deux diplômes qui s'obtiennent par deux ans de formation après le baccalauréat. Maillard, Veneau et Grandgérard (2004, p. 20) distinguent 4 manières de situer les LP par rapport aux formations de DUT et BTS : un approfondissement année de licence générale sont très peu nombreux à intégrer le dispositif de formation alors que l'on peut raisonnablement penser qu'ils ne sont pas tous destinés à se diriger vers un master (Combes, 2011, p. 28).

Il faut noter que les LP ont été (et sont encore) portées majoritairement par des enseignants de DUT et/ ou de $\mathrm{DU}^{9}$, dispositifs où professionnalisation et alternance étaient déjà mises en œuvre au moment de la création du diplôme, et où les liens avec les professionnels du domaine existaient déjà et ont été, dans ce nouveau dispositif, resserrés. Pour les autres responsables, appartenant notamment aux UFR (Unités de formation et de recherche, disciplinaires), l'expérience de la professionnalisation était historiquement moins développée. Pour autant, une volonté de diversifier l'offre de formation au niveau bac +3 dans leur filière et de s'investir dans la formation professionnelle a poussé plusieurs d'entre eux à créer des LP au sein de leur composante. Ces créations ex nihilo ont plutôt reposé, selon Maillard, Veneau et Grandgérard (2004, p.63), sur des relations avec des représentants d'organismes de formation, de chambres de commerce et non pas avec des employeurs en direct. Charge ensuite aux étudiants, lors de leur recherche de stage, de développer eux-mêmes des relations avec les milieux professionnels. Les auteurs identifient une troisième configuration radicalement différente des deux précédentes ayant été à la source de créations de LP : il s'agit des offres impulsées de l'extérieur de l'université. C'est notamment par exemple le cas dans le secteur bancaire où les acteurs économiques se sont adressés aux universitaires pour qu'ils acceptent de créer un dispositif de formation correspondant exactement à leurs besoins. Les universitaires sont, dans ce cas précis, dans une configuration qui les dépossède largement de leur prérogative, qu'il s'agisse de la conception du dispositif ou de ses modalités d'évaluation.

La prescription d'une construction d'un partenariat avec le monde économique au niveau de la conception des projets de création de LP a permis de répondre « de manière ciblée à une demande locale de formation professionnelle de haut niveau» (Giret, 2008, p. 59). Les emplois visés à l'issue des LP correspondent théoriquement au niveau de qualification de

aspect déjà vu), une intégration (mise en commun avec une autre spécialité) ou encore un élargissement (décloisonnement sectoriel, fonctionnel).

9 Le DU (Diplôme universitaire) est un diplôme propre à une université qui ne rentre pas dans le système LMD. 
cadre intermédiaire et sont en rapport avec la formation suivie. Répondant à des besoins en compétences bien identifiés, les LP sont reconnues et appréciées des employeurs, avec un taux moyen d'insertion des diplômés avoisinant les $91 \%{ }^{10}$. Pour autant, Giret (2008, p.63) nuance ces résultats en pointant le fait que seulement $17 \%$ d'entre eux accèdent directement à un emploi de cadre à la sortie de la formation.

II reste que ce diplôme constitue «la forme la plus poussée d'impliquer les professionnels dans la formation » (Maillard \& Veneau, 2006, p.63), non seulement dans la phase de construction et dans I'habilitation des dossiers au niveau de la commission nationale d'expertise (où universitaires et professionnels siègent à parité) mais aussi dans la phase de mise en œuvre en tant que telle de la formation à l'occasion des enseignements professionnels et de l'alternance. Les LP rassemblent plus d'un tiers des apprentis de l'université et, dans la version stage, les étudiants sont en moyenne durant 16 semaines en milieu professionnel. C'est sur cette caractéristique du diplôme que se centrent les analyses présentées ci-dessous basées sur des entretiens avec des responsables universitaires de LP.

\section{Méthodologie de recueil et d'analyse des données}

Les données de recherche ici mobilisées ont été recueillies dans le cadre d'entretiens semi-directifs structurés avec des responsables de LP. Ces entretiens se sont déroulés selon un schéma préalablement établi regroupant une grille de thèmes et de sous-thèmes :

- statut des étudiants dans la formation;

- relations avec les milieux professionnels (naissance de la formation, métiers visés par le diplôme, nature des partenariats avec les professionnels...);

- organisation pédagogique de l'alternance (recherche des terrains, rythme, articulations fonctionnelles entre université et entreprises, évaluation...);

- insertion;

- référentiel de compétences, jurys VAE.

Ce canevas a été établi dans l'objectif de balayer les volets principaux de la professionnalisation du point de vue des acteurs universitaires.

Les LP retenues sont sur plusieurs sites universitaires dans une même région (Bourgogne, Franche-

10 Enquête sur l'insertion professionnelle des diplômés 2010 de I'université, menée par le ministère de l'Enseignement supérieur et de la Recherche.
Comté). Nous en avons sélectionné 23 sur un total de 80 selon les caractéristiques suivantes : représentativité des composantes (avec une proportion plus grande des IUT par rapport aux UFR, à savoir respectivement $2 / 3$ et $1 / 3$ ), représentativité de la diversité des statuts des étudiants (formation initiale, formation continue, alternants ou stagiaires). L'échantillon a également été construit en veillant à ce qu'il soit significatif des différentes spécialités dans lesquelles les LP ont vu le jour et qui sont par ordre décroissant : droit, économie, gestion (11), sciences, technologie, santé (8), sciences humaines et sociales (3), sciences et techniques des activités physiques et sportives (1) 11 . On compte actuellement une offre de plus de 2000 LP sur le territoire français. Notre travail d'investigation, réalisé sur 23 d'entre elles, se situe à un échelon local et ne vise pas une représentativité mais l'apport d'un éclairage sur des pratiques en évolution.

Les verbatim ont ensuite été intégrés dans le logiciel Alceste (Analyse des Lexèmes Cooccurents dans un Ensemble de Segments de Texte). Celui-ci est un outil d'analyse statistique des données textuelles qui établit des classes de vocabulaire ou "mondes lexicaux», chacune des classes étant constituée de mots s'y rapportant. Le recours à Alceste "entraîne une systématisation et une objectivation de l'analyse textuelle, qui tend à réduire le poids des attributions préconçues du chercheur dans la définition des unités d'analyse, par rapport à un traitement manuel» (Bart \& Fournet, 2010). Le travail d'interprétation repose sur la recherche de la cohérence interne des mots de chaque classe. La possibilité d'accéder aisément aux extraits de phrases dans lesquels est placé le vocabulaire constitutif de chaque classe permet d'enrichir l'analyse en tenant compte du contexte dans lequel les mots sont mobilisés par les locuteurs. Nous nous centrons sur deux univers lexicaux pour lesquels nous croisons les sous-classes qui les constituent avec une analyse thématique transversale en lien avec notre problématique de la coupure et du lien dans la gestion des LP en alternance. Plus précisément, nous identifions comment les univers lexicaux et leurs sous-thèmes proposés par Alceste donnent des indications sur la manière dont les responsables pédagogiques pensent et organisent les liens entre les différents univers : quels sont les registres pour lesquels les articulations entre monde éducatif et monde productif

11 Au niveau national, les LP en sciences et technologie dominent, suivies à égalité par droit, sciences économiques et lettres, sciences humaines. 
Tableau 1. Classes et sous-thèmes lexicaux des différentes modalités d'articulation

\begin{tabular}{l|l|l}
\hline Ce qui fait lien & Ce qui fait tension & Ce qui fait coupure \\
\hline \multicolumn{2}{l|}{ Classe 2. L'alternance dans ses dimensions organisationnelles et temporelles } & $\begin{array}{l}\text { Classe 3. L'alternance dans ses } \\
\text { dimensions évaluatives }\end{array}$ \\
\hline $\begin{array}{l}\text { Sous-thème 1. Articulation statut et } \\
\text { alternance }\end{array}$ & $\begin{array}{l}\text { Sous-thèmes 3 et 4. Découpage des } \\
\text { périodes }\end{array}$ & $\begin{array}{l}\text { Sous-thème 1. L'évaluation de } \\
\text { l'étudiant en situation } \\
\text { professionnelle } \\
\text { Sous-thème 2. La production du } \\
\text { rapport de stage } \\
\text { et emploi }\end{array}$ \\
\begin{tabular}{l} 
Sous-thème 3. La soutenance \\
\hline
\end{tabular}
\end{tabular}

se font et quels sont ceux pour lesquels des tensions mais aussi des coupures se donnent à voir? Des extraits de citations des entretiens avec les responsables des LP sont sélectionnés à partir de leur indice de représentativité dans leur sous-classe.

\section{Les résultats et leurs interprétations}

Dans un premier temps, nous rendons compte des univers lexicaux proposés par le logiciel avant de nous centrer sur les modes d'articulation entre le monde académique et universitaire. Le rapport proposé fait état de 2548 Unités de contexte élémentaires (UCE) classées, ce qui correspond à $74 \%$ de la totalité des unités. La variable liée aux composantes différentes dans lesquelles les LP sont proposées (UFR ou IUT) a été contrôlée : elle ne joue aucun rôle dans la constitution des classes. Les UCE se répartissent en 4 classes stables.

La classe 1 qui représente $39 \%$ des unités textuelles est la première à s'être dégagée dans l'arbre de classification, c'est aussi la plus homogène (1 004 unités). Elle se caractérise par des mots tels que : métier, social, tourisme, besoin, fromager, international... Elle recouvre l'univers lexical afférent aux différents domaines de formation et professionnels dans lesquels les LP faisant partie de l'échantillon se situent. Cette classe reflète les liens étroits - déjà évoqués précédemment - entre les acteurs de l'université et les acteurs du monde socio-économique, liens fondés sur «une stratégie de niches » (Combes, 2011, p.28) ayant conduit les universitaires à proposer un très grand nombre de LP lors de la création du diplôme ou dans les années qui ont suivi.

Ensuite se détache la classe 2 qui représente $32 \%$ des unités textuelles (826 unités analysées). Ses mots significatifs sont : année, contrat, entreprise, semaines, de l'alternance, notamment dans ses dimensions organisationnelles et temporelles. Nous développons le contenu de cette classe plus en avant dans le texte.

Elle est suivie de la classe 3 (511 unités) qui représente $20 \%$ des unités textuelles et dont les mots significatifs sont : évaluation, tuteur, notes, soutenances, rapport... Elle recouvre l'univers lexical de l'évaluation spécifique aux LP distribuée entre universitaires et professionnels. Nous exposons également le contenu de cette classe dans la suite de notre écrit.

Enfin émerge la classe 4 (205 unités) représentant $9 \%$ des unités textuelles et constituée par les mots tels que candidats, sélection, validation, pré (inscription ou recrutement)... Elle renvoie aux modalités et priorités données dans l'étape de sélection des étudiants mais ne sera pas exploitée, eu égard à la distance avec la problématique ici développée.

Les modes d'articulation entre les deux univers s'analysent autour de ce qui fait lien, tension et coupure (voir tableau 1).

Deux sous-thèmes de la classe 2 portant sur l'alternance dans ses dimensions organisationnelles et temporelles permettent d'identifier ce qui fait lien entre les acteurs du monde académique et ceux du monde économique : I'un est centré sur la mobilisation des milieux professionnels durant la formation, l'autre sur l'insertion, et plus globalement, sur l'accès des étudiants à des emplois de qualité.

On identifie dans les verbatim trois modalités qui se déclinent égalitairement dans le fait d'accéder à un terrain (sous forme de contrat ou de simple convention) : la première dans laquelle l'étudiant est en demeure de "trouver par lui-même», la seconde où il peut prendre appui sur un listing fourni par la composante et la troisième où un nombre d'étudiants accueillis est défini avec les entreprises partenaires, le plus souvent par la voie d'un contrat en alternance. 
La proximité des membres de l'équipe pédagogique avec les acteurs des lieux d'accueil potentiels pour les étudiants est recherchée, qu'ils soient au service ressources humaines ou professionnelles du domaine :

Nous on a besoin plus de faire de la proximité, de discuter, d'appeler les patrons de petites entreprises en sous-traitance et on essaie de jouer sur ce terrain-là (LP production industrielle, spécialité conception et production aéronautique).

Dans notre échantillon, les relations soutenues se font majoritairement avec les entreprises de taille moyenne ou petite. Il est souligné, dans ces lieux-là, la possibilité d'avoir des relations de proximité dans la durée alors que pour les grands groupes, les interlocuteurs changent. Pour les licences ayant au moins 5 années d'ancienneté, l'exemple est donné d'anciens étudiants embauchés dans des entreprises du domaine qui deviennent, à leur tour, des tuteurs. C'est aussi en se rendant sur les salons professionnels que les contacts avec les entreprises, lieux d'accueil potentiels, peuvent se nouer.

Le sous-thème "Alternance et emploi » condense la préoccupation des responsables pédagogiques pour l'accès à l'emploi de leurs étudiants à l'issue de la formation :

Après beaucoup d'entreprises jouent le jeu, ce que j'ai vu l'année dernière, après ils les gardent les jeunes. Beaucoup l'année dernière ont reçu des propositions pour être embauchés. Ils prennent des apprentis pour les recruter à la fin (LP bâtiment et construction, spécialité conducteur de travaux en maisons individuelles).

Moi mon objectif, c'est que les jeunes ils soient embauchés à la fin donc on essaie vraiment d'avoir de très bonnes relations avec eux [les entreprises] (LP commerce, spécialité distribution, mention management et gestion de rayons).

Les responsables du diplôme sont dépendants des choix des entreprises en matière de recrutement, ils tentent d'en discerner les stratégies et de soigner le relationnel. Le souci de l'insertion est en lien avec l'employabilité des étudiants à l'issue de la formation, souci qu'une responsable de LP résume ainsi :

Nous toujours le souci ça a été la professionnalisation en disant en 21 semaines, qu'est-ce qu'ils doivent savoir, acquérir pour être employable? (LP attaché au développement international des organisations).

Les membres des équipes pédagogiques sont en veille pour identifier des connaissances et/ou des compétences à intégrer par leurs étudiants qui sont requises dans les métiers pour lesquels ils les forment.
Dans la plupart des entretiens à notre disposition, les responsables mettent en avant la participation de professionnels à la fois dans le réajustement des contenus de formation et dans la transmission même de ceux-ci.

Les tensions entre monde académique et économique se donnent à voir au niveau des sous-thèmes portant sur le découpage des périodes.

Deux grandes options se dégagent sur la question du rythme de l'alternance : soit le temps sur le terrain est regroupé sur le second semestre, soit il est échelonné tout au long de l'année universitaire et il prend alors une grande variété de formes (un mois/un mois, trois semaines/trois semaines, une semaine/deux ou trois semaines, trois jours/deux jours...). Le rythme de l'alternance est aussi pensé en fonction de l'éloignement géographique des étudiants, éloignement de leur lieu de résidence, éloignement entre l'université et l'entreprise. Des voyages trop nombreux génèrent un coût difficilement supportable pour l'étudiant sans compter qu'il doit financer un double logement, c'est là une autre préoccupation des responsables de formation lorsqu'ils organisent l'alternance.

Le choix des rythmes relève d'une recherche de la meilleure articulation possible entre les deux univers :

- soit en lien avec une progression pédagogique, progression organisant une responsabilisation grandissante de l'étudiant au fil des mois :

Les apprentis suivent des séances de cours suivies de périodes de formation en magasin de 3 à 10 semaines. La durée des périodes augmente à mesure que l'on avance dans le cycle de formation (LP logistique de distribution et transports internationaux).

- soit en lien avec la nature de l'activité professionnelle. Dans la LP responsable d'ateliers de production fromagère, la planification des temps sur le terrain a été pensée au regard des attentes des professionnels :

Pour être tout à fait clair, on a répondu aux contraintes de la production laitière que nous ont indiquées les professionnels en nous disant, nous quand les vaches sont au champ au mois de mai, on est débordés de boulot et on a besoin des apprentis et des stagiaires dans les ateliers, car c'est de la main-d'œuvre, il faut quand même le savoir, et on ne peut pas les laisser en formation en mai, juin sur ces périodes qui sont cruciales pour nous dans la production.

Entre les deux préoccupations, celles de la pédagogie et celle de la production, des tensions émergent régulièrement :

Ça a été un petit peu un combat car ils [les professionnels] étaient habitués à prendre des apprentis qui avaient moins de semaines de cours donc là on leur 
a dit attention vous avez des gens qui sont d'un autre niveau de formation, qui leur coûtent un peu plus cher en apprentissage parce qu'ils sont plus âgés que ceux qu'ils avaient auparavant mais d'un autre côté, ils s'y retrouvent car ils ont des gens qui sont plus autonomes et qui peuvent travailler avant $6 \mathrm{~h}$ du matin (LP responsable d'ateliers de production fromagère).

On peut penser dans cette situation que les négociations avec les professionnels ont été facilitées par la très bonne connaissance que le responsable de la LP possède du terrain. Enfant du pays, il déclare avoir « toujours maintenu des liens étroits avec le milieu agricole et les filières agroalimentaires régionales » tout en développant des travaux de recherche autour du concept de terroir et plus précisément sur l'identité géographique d'un produit en tant que facteur de développement local. Au moment de l'entretien, il réalisait un diagnostic sur les besoins de la filière en formation continue basé sur environ 90 interviews avec des professionnels de la transformation laitière.

L'issue de ces tensions prend appui sur des réglages successifs, à partir de l'expérience :

Au début on faisait un mois/un mois, et les entreprises nous ont dit, non ça va pas, on les perd pendant le mois où ils sont chez vous, ils ne sont plus dans le bain quand ils reviennent (LP commerce, spécialité commerce des vins et œnotourisme).

On a changé ce découpage cette année par rapport à ce qui se faisait l'année précédente [...] ils étaient trop éloignés de l'entreprise longtemps, et même pour les va-et-vient au niveau des cours, parce que ce qui est très intéressant au niveau des cours, c'est de suivre ce qu'ils font en entreprise (LP logistique de distribution et transports internationaux).

Et puis que l'étudiant il soit aussi là parce que l'alternance, elle a un problème, c'est qu'à un moment donné, il est plus souvent dans l'entreprise qu'à l'université et il en oublie l'importance universitaire, venir à l'université pour lui, c'est quasi les vacances sauf qu'on va lui demander de travailler, d'être évalué et on le remet dans un statut particulier qui est celui d'étudiant donc il passe du statut de l'employé au statut d'étudiant, pour gérer c'est pas simple (LP électricité et électronique, spécialité véhicules, électronique et gestion des automatismes).

Ces extraits de verbatim illustrent clairement la ressource que constitue l'alternance d'un point de vue pédagogique mais aussi sa principale difficulté qui voit s'enchaîner des périodes aux activités très différentes impliquant des préoccupations et des rôles eux aussi différents. Psychiquement, il s'agit pour les alternants de se mettre en phase avec les attentes des différents comme les professionnels sont bien conscients que les transitions génèrent des ruptures et nécessitent une adaptation de l'étudiant à chaque changement. Dans l'entretien pour la LP électricité et électronique, les universitaires ont fait longuement état de leurs difficultés d'une part «à les remettre sur les rails» à leur retour d'entreprise pour poursuivre un enseignement débuté dans la période précédente et d'autre part à les sortir des schémas conventionnels d'enseignement : faire travailler en autonomie leurs étudiants dans le cadre d'un projet tutoré, sans la présence d'enseignants, s'avère périlleux tant les étudiants «reprennent des habitudes d'apprenants lambda, scolaires » à leur retour en formation. Les difficultés sont donc tout autant dans l'exercice mental que dans la posture requise.

C'est dans l'univers lexical de la classe 3, fortement attaché à la question des modes d'évaluation des étudiants que s'identifie principalement ce qui fait coupure entre les deux univers. Les formes de coopération à ce sujet entre professionnels et enseignants se révèlent peu étroites, voire impossibles. D'un point de vue formel, trois temps distincts renvoyant aux trois sous-thèmes proposés par Alceste constituent des modes d'évaluation liés à l'alternance. Ils s'ajoutent aux modes d'évaluation des différentes unités d'enseignements qui eux-mêmes sont fortement structurés autour d'études de cas, d'exposés individuels ou collectifs et privilégient le contrôle continu aux examens terminaux. II s'agit de l'évaluation de l'étudiant en situation professionnelle, de la production du rapport de stage (ou mémoire) et de la soutenance.

Pour ce qui concerne l'évaluation de l'étudiant en situation professionnelle, on constate que la place prise et/ou laissée aux tuteurs professionnels dans les évaluations terminales est variable : tous les tuteurs ont à leur disposition des grilles avec différents critères pour apprécier l'activité de l'étudiant mais la transformation en note ainsi que l'importance qui lui est attribuée donnent à voir une très grande hétérogénéité :

Il y a une grille qui est remplie par l'entreprise et de là on extrait une note, on l'intègre à hauteur de, ça va être dans les $50,60 \%$ de la note finale. On n'a pas voulu non plus, c'est toujours un peu pareil, les entreprises souvent ont tendance à mettre des notes qui sont pas trop mauvaises parce que souvent elles ont l'impression de se noter elles-mêmes, les tuteurs en tous les cas, donc on hésite à donner trop d'importance aux notes de l'entreprise. Mais là elles ont quand même une part importante dans l'évaluation, c'est plus de la moitié, mais nous aussi on a une note sur la visite, on note la visite et là aussi dans la visite, il y 
a une grille qui est détaillée avec plusieurs paramètres (LP production industrielle, spécialité conception 3D avancée et calculs de structures).

Pour contourner l'obstacle de la notation, il peut être demandé aux tuteurs une appréciation sur le déroulement du stage :

[...] en fait on leur demande pas de mettre une note, on leur donne une feuille avec des critères $A B C D E$, et on leur demande une appréciation avec les critères et, à partir de là, ça nous donne une note entreprise. Parce que c'est très difficile, demandez à un maître de stage de mettre un 12 , un 14 , un 18 , déjà que nous c'est pas évident, lui il en sait rien, donc si vous lui dites des critères, je sais pas, esprit d'initiative je dis n'importe quoi, $A B C D E$, E la plus mauvaise, il va vous évaluer à partir de là et ça nous permet de normaliser ça. Alors la grille d'évaluation est peut-être pas parfaite mais au moins elle existe donc globalement quand ils sont pas satisfaits de l'étudiant, on voit que les croix vont toutes du même côté, le maître de stage a passé 10 semaines avec un stagiaire c'est parfois pas facile d'évaluer un jeune avec qui on a créé des affinités (LP production industrielle, spécialité conception et production aéronautique).

Bien qu'appartenant à un même champ de formation, la production industrielle, les deux responsables qui s'expriment ainsi sont impliqués dans des activités sur des sites différents, dans des structures différentes (UFR ou IUT) et ont des partenaires eux aussi différents. Pour autant, ils exemplarisent les propos liés au contenu de cette classe, sur la complexité d'une activité fondamentale d'un enseignant universitaire, l'évaluation, notamment lorsqu'elle s'applique à des apprentissages qui se déroulent hors centre, dans le milieu professionnel. Les responsables remarquent ainsi que souvent les professionnels attribuent des notes qui feraient fi de l'objectivité requise : parce qu'ils se sont fortement impliqués dans le suivi, qu'ils ont des affinités avec l'étudiant, parce qu'ils ont l'impression de se «noter eux-mêmes», ils donneraient des notes trop élevées ou sans rapport avec les règles docimologiques universitaires. Aux fins d'homogénéiser le système, un certain nombre de responsables ont pris des mesures telles celles données en exemple ci-dessus et ce sont eux qui déduisent ensuite une note. Car, comme le remarque l'un d'entre eux, c'est l'enseignant tuteur qui a «finalement toutes les cartes en main pour l'évaluation, c'est lui qui est au cœur du processus » (LP ressources humaines petites et moyennes entreprises).

La production du rapport de stage renvoie quant à elle à des critères académiques (qualité de l'écriture, mise en forme, références, recherches documentaires...) mais aussi à des capacités d'analyse :
Vous êtes acteur de votre formation, donc un des moyens pour être acteur de votre formation, un des outils ça va être votre rapport de stage, et le rapport de stage, ça va pas être une description des tâches, ça va être une analyse des compétences qui ont été nécessaires pour réaliser les activités qu'on m'a demandées [...] c'est-à-dire que chaque rapport de stage, ça consiste à faire un diagnostic sur la rencontre ou non entre les situations d'entreprises dans lesquelles ils sont et ce que leur a apporté ou non la licence, et de proposer quelque chose (LP hôtellerie et tourisme, spécialité gestion touristique territoriale et internationale).

Ces attendus sont de même nature en ce qui concerne l'évaluation des projets tutorés : les enseignants insistent également sur le fait qu'il ne s'agit pas d'un rapport «dans lequel on dit ce qui s'est passé », ni d'un «mémoire», pour bien montrer «qu'on étudie un problème». II reste que l'on est ici dans une composante de l'évaluation prise en charge de façon exclusive par la communauté universitaire qui en fixe les attentes et procède à la notation. On retrouve d'ailleurs dans les attentes exprimées la caractéristique fondamentale de la posture épistémologique universitaire telle que Romainville l'a résumée : «le discours académique privilégie l'analyse et la critique; la narration et la description n'y sont pas valorisées» (2000, p. 40).

Enfin, la soutenance est un moment privilégié pour accueillir les professionnels, et leur présence est fortement encouragée pour les étudiants ayant un statut de stagiaire (elle est systématique pour les étudiants sous contrat). Elle offre l'occasion de réunir étudiants, enseignants et tuteurs pour un dialogue portant sur des éléments de bilan. Elle produit une note supplémentaire à celle de l'expérience professionnelle ellemême et à celle du rapport écrit.

Si les points de vue croisés sont qualifiés d'incontournables dans cet exercice, là encore la place effective donnée aux tuteurs professionnels est relative puisqu'ils peuvent être membres du jury, être interpellés pour donner leur appréciation mais sans être pour autant décideurs au même titre que l'enseignant de la note attribuée. Les enseignants peuvent d'ailleurs définir des critères d'évaluation relevant de leur unique champ de compétence :

Maintenant il y a aussi la partie soutenance, c'est plus académique et il y a une évaluation qui est faite sur la compétence à communiquer dans un temps limité, donc ça, c'est nous qui gérons (LP production industrielle, spécialité conception 3D avancée et calculs de structures). 
Il est important de souligner que ces constats s'appliquent pour toutes les LP de notre échantillon, sauf pour la LP banque finances assurance. Celle-ci, comme nous l'avons indiqué précédemment, a été mise en place à la suite d'une demande de la branche professionnelle et l'organisme de formation interne prend en charge des pans entiers de la formation et de l'évaluation, en parallèle des activités universitaires.

\section{Discussion et perspectives}

Plusieurs résultats peuvent être soulignés dans notre étude portant sur la conception et les modes de réalisation de l'ouverture des responsables pédagogiques de LP au monde professionnel notamment sur le plan de l'alternance.

On observe une véritable intention d'articuler les deux mondes dans lesquels les étudiants évoluent. Cette articulation renvoie à une attention particulière portée aux dimensions relationnelles et organisationnelles. Ce qui fait lien se réalise en particulier dans des constructions partenariales établies dans la proximité et si possible dans la durée afin de maximiser la relation entre formation et emploi. Les responsables pédagogiques consacrent ainsi une part importante de leur activité à entretenir «un réseau de relations instituantes» (Geay et al., 1999, p. 114) avec différents acteurs des entreprises tout en étant conscients de la dimension éphémère de celui-ci. Si ces liens semblent s'organiser plutôt dans une relation asymétrique - les universitaires étant dépendants des choix des entreprises à la fois en matière d'accueil de stagiaires (ou d'alternants) mais aussi de recrutement -, ceux-ci s'équilibrent par le besoin des acteurs professionnels en matière de recrutement de cadres intermédiaires.

L'alternance nécessite de la part de ses concepteurs une adaptabilité importante : non seulement ils ne peuvent plus avoir comme modèle de conduite unique la diffusion de savoirs dont ils auraient seuls délimité le périmètre - une place doit être faite à l'employabilité et les compétences qui s'y rapportent mais ils doivent composer sur un plan organisationnel avec les milieux professionnels. Les résultats de la recherche soulignent les ajustements successifs au niveau des temps passés sur le terrain ou à l'université, fruits de la résolution de tensions perçues au travers du comportement des étudiants ou des échanges avec les professionnels. Ainsi, la rythmicité est pensée à la fois pour correspondre à la nature de l'activité profes- physiquement et mentalement à l'université. Même si I'on est loin d'une conception «juxtapositive», tout se passe comme si une sorte de concurrence s'établissait entre les deux mondes, soit que les étudiants ne sont plus «dans le bain » professionnel à leur retour de l'université, soit que celle-ci est perçue comme «des vacances", après la période passée dans l'entreprise. Chacun des univers chercherait à tirer la couverture à soi, les entreprises plutôt à la recherche d'une maind'œuvre productive à des moments clés de leur activité et les universitaires plutôt préoccupés d'avoir des étudiants investis dans le hic et nunc du programme pédagogique. On peut penser que I'on rencontre ici les limites de l'alternance telles qu'elles étaient déjà identifiées par Geay et alii, (1999, p. 113) lorsqu'ils déploraient l'absence de mariage étroit pour les apprenants sur un plan cognitif entre les lieux d'apprentissage dans l'alternance.

La difficulté de l'alternance intégrative apparaît également saillante dans la conception de l'évaluation des acquis de l'expérience de terrain. On touche ici une prérogative importante du métier d'enseignant, à savoir non seulement évaluer mais attribuer une note. La confiance accordée aux tuteurs professionnels pour la notation est toute relative. La suspicion d'une notation empreinte d'une relation de proximité avec l'étudiant et donc éloignée d'une notation qui se voudrait objective est pointée. Normalisation et critères académiques viennent faire barrage en quelque sorte aux appréciations des tuteurs professionnels.

Nos résultats de recherche vont dans le même sens que ceux développés par Maillard et Veneau lorsqu'ils écrivent que «même si la licence professionnelle a été l'occasion de resserrer les liens entre enseignants et professionnels ou d'en créer, ces derniers relèvent toujours d'une répartition des rôles assez traditionnelle, assez éloignée de l'idéal du "partenariat" tel qu'il est esquissé dans "les discours volontaristes de la professionnalisation"»(2006, p.66).

Pour que l'activité d'évaluation mais aussi plus globalement d'ingénierie dans les dispositifs de formation en alternance puisse poursuivre son développement, nous rejoignons d'autres auteurs et notamment Astier (2007, p. 61-62) qui pointe la nécessité de construire des "artefacts» à fonction de référence : par exemple les référentiels de formation ou encore les référentiels métiers. Dans cette perspective, les référentiels sont moins vus comme une manière de répondre d'une seule voix aux questions qui se posent que comme moyen de rendre véritablement discutables les critères 
d'une évaluation et par là même la nature des savoirs et compétences à acquérir. Par exemple, dans les LP qui constituent notre échantillon, une compétence apparaissait centrale dans les attentes, celle de la problématisation entendue comme une capacité à favoriser l'interrogation sur les fondements de l'action engagée à partir de différents points de vue et la formulation de différentes alternatives. Cette attente n'est pas formellement énoncée par les responsables de LP interviewés mais ils la décrivent de manière indirecte. Ils vont formuler la nécessité pour les étudiants «qu'ils prennent du recul», qu'ils acquièrent des «savoirs de portée intermédiaire», savoirs qu'ils distinguent de ceux renvoyant à une application directe et qu'ils lient à une posture spécifique relevant de la «réflexivité». Professionnels et équipes pédagogiques pourraient définir ensemble un certain nombre de situations pédagogiques à fort «potentiel d'apprentissage et de développement » (Mayen, 2007, p. 86) où cette compétence serait susceptible d'être acquise par les étudiants ainsi que les critères permettant de l'évaluer.

Des espaces de débat sont à créer pour instruire les conditions de la mise en œuvre d'un processus de

\section{Bibliographie}

AFPA, CEE, CNAM \& INRP (1992). Les formations en alternance. Paris : La Documentation française.

ASTIER P. (2007). «Alternance construite, prescrite, vécue». Éducation permanente, $\mathrm{n}^{\circ} 172$, p. 61-72.

BART D. \& FOURNET M. (2010). «Le projet professionnel et personnel des étudiants, assises de leur professionnalisation?». Revue internationale de pédagogie de l'enseignement supérieur, $\mathrm{n}^{\circ} 26-1$. En ligne : <http://ripes.revues. org/314> (consulté le 18 avril 2016).

BOURGEON G. (1979). Socio-pédagogie de l'alternance. Paris : UNMFREO.

CHAIX M.-L. (1993). Se former en alternance. Paris : L'Harmattan.

CHAUVIGNÉ C. \& COULET J.-C. (2010). «L'approche par les compétences : un nouveau paradigme pour la pédagogie universitaire?». Revue française de pédagogie, $\mathrm{n}^{\circ} 172$, p.15-28. En ligne : <http://rfp.revues.org/2169> (consulté le 20 mars 2017).

COMBES M.-C. (2011). «La professionnalisation des études universitaires ». Éducation permanente, n 188, p. 25-35.

GEAY A. et al. (1999). «Actualités de l'alternance». Revue française de pédagogie, $\mathrm{n}^{\circ} 128, \mathrm{p} .107-125$.

GIRET J.-F. (2008). «Le mouvement de professionnalisation des études supérieures, l'exemple des licences professionnelles ». Relief Céreq, n²5, p.59-62. professionnalisation de qualité. L'objectif n'est pas de parler d'une seule voix ni de se partager des zones de pouvoir et d'influence mais d'articuler les différents mondes sans pour autant les fusionner. Avancer cette conclusion revient à proposer que la professionnalisation soit conçue comme une activité dont la dynamique même est constituée par sa conflictualité (Henry \& Bournel-Bosson, 2014) autour de questions communes : comment se définit la professionnalité dans un champ d'activité ciblé? Comment l'étudiant peut-il devenir pleinement acteur de son parcours de professionnalisation en «permettant à chacun d'assumer la variété des situations dans I'unité de son milieu » (Geay et al., 1999, p. 120)?

Maryse Bournel-Bosson Université de Bourgogne Franche-Comté, laboratoire de psychologie (EA 3188), IUT Belfort, département Carrières sociales maryse.bournel-bosson@univ-fcomte.fr

Muriel Henry

Université de Bourgogne, pôle Formation et Vie universitaire muriel.henry@u-bourgogne.fr

HENRY M. \& BOURNEL-BOSSON M. (2014). «La professionnalisation dans l'enseignement universitaire : un processus dialogique? ». Revue internationale de pédagogie de l'enseignement supérieur, n³0-2. En ligne : <http://ripes. revues.org/817> (consulté le 3 juillet 2017).

LEMISTRE P. (2015). «La professionnalisation des formations initiales : une solution aux mutations du marché de l'emploi pour les jeunes?». Revue française de pédagogie, n¹92, p.61-72.

LERBET-SERÉNI F. (1997). «Analyse de pratiques et pratiques de l'alternance en formation de formateurs ». In D. Violet (dir.), Formations d'enseignants et alternances. Paris : L'Harmattan, p.137-174.

LICHTENBERGER Y. (1995). «Alternance en formation et qualification professionnelle». In R. Poupard, Y. Lichtenberger, J.-M. Luttringer \& C. Merlin, Construire la formation professionnelle en alternance. Paris : Éditions d'Organisation.

MAILLARD D., VENEAU P. \& GRANDGÉRARD C. (2004). «Les licences professionnelles. Quelle acceptation de la "professionnalisation" à l'université? ». Relief Céreq, n5.

MAILLARD D. \& VENEAU P. (2006). «Les licences professionnelles. Formes et sens pluriels de la professionnalisation à l'université ». Sociétés contemporaines, n 62, p.49-68.

MALGLAIVE G. (1994). «Alternance et compétences». Les Cahiers pédagogiques, $\mathrm{n}^{\circ} 320$, p. 26-28. 
MAYEN P. (2007). «Passer du principe d'alternance à l'usage de l'expérience en situation de travail comme moyen de formation et de professionnalisation». In F. Merhan, C. Ronveaux \& S. Vanhulle (dir.), Alternances en formation. Bruxelles : De Boeck, p. 83-100.

PATROUCHEVA M. \& TRIBY E. (2010). «Alternance et gouvernance, le devenir de la professionnalisation à l'université». Colloque international Les Stages et leur gouvernance en débats, organisé à l'université Paris-Est-Créteil, les 18 et 19 juin 2010.

ROMAINVILLE M. (2000). L'échec dans l'université de masse. Paris : L'Harmattan.

ROSE J. (2008). «La professionnalisation des études supérieures. Tendances, acteurs et formes concrètes». Relief, $n^{\circ} 25$ : «Les chemins de la formation vers l'emploi : $1^{\text {re }}$ biennale Formation-emploi-travail», p.43-58.
ROUCOULES A. (2004). «L'alternance, de la formation à l'exercice professionnel». Empan, n56, p. 137-142.

ULMANN A.-L. (2012). «L'alternance à l'université à l'épreuve de ses limites ». Éducation permanente, n¹93, p.163-172.

VANHULLE S., MOTTIER-LOPEZ L \& DEUM M. (2007). «La co-construction de soi et de ses savoirs professionnels comme effet de l'alternance : quels indicateurs?». In F. Merhan, C. Ronveaux \& S. Vanhulle (dir.), Alternances en formation. Bruxelles : De Boeck, p. 241-257.

WITTORSKI R. (2012). «La professionnalisation de l'offre de formation universitaire : quelques spécificités». Revue internationale de pédagogie de l'enseignement supérieur, n²8-1. En ligne : <http://ripes.revues.org/580> (consulté le 16 octobre 2015). 